urgent need for further studies into the mucosal immune responses and microbiota during infection with SARS-CoV-2 for better understanding of the disease progression and local mechanisms that are important for either swift clearance of infection with only mild symptoms or the prevention of tissue damage.

Simon P. Jochems (DD $1 \bowtie$,

Daniela M. Ferreira (D) ${ }^{2}$ and

Hermelijn H. Smits (D)

${ }^{1}$ Department of Parasitology, Leiden University

Medical Center, Leiden, the Netherlands.
${ }^{2}$ Department of Clinical Sciences, Liverpool School of

Tropical Medicine, Liverpool, UK.

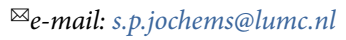

Published online: 21 October 2021

https://doi.org/10.1038/s41590-021-01041-w

$\neg$ References

1. Smith, N. et al. Nat. Immunol. https://doi.org/10.1038/s41590021-01028-7 (2021).

2. Cervia, C. et al. J. Allergy Clin. Immunol. 147, 545-557.e549 (2021).

3. Hadjadj, J. et al. Science 369, 718-724 (2020).

4. Lei, X. et al. Nat. Commun. 11, 3810 (2020).

5. Ziegler, C. G. K. et al. Cell 184, 4713-4733.e22 (2021).

6. Carniel, B. F. et al. JCI Insight 6, el41088 (2021).
7. Hoepel, W. et al. Sci. Transl. Med. https://doi.org/10.1126/ scitranslmed.abf8654 (2021).

8. Gupta, A. et al. Microbes Infect. https://doi.org/10.1016/j.micinf. 2021.104880 (2021).

9. Schaupp, L. et al. Cell 181, 1080-1096.e19 (2020).

10. Arunachalam, P. S. et al. Science 369, 1210-1220 (2020).

11. Mitsi, E. et al. Preprint at medRxiv https://doi.org/10.1101/ 2021.07.22.21260837 (2021).

12. Lewnard, J.A. et al. J. Infect. Dis. https://doi.org/10.1093/infdis/ jiab128 (2021).

13. Lehtinen, M. J. et al. Sci. Rep. 8, 11411 (2018).

14. de Steenhuijsen Piters, W. A. et al. Am. J. Respir. Crit. Care Med. 194, 1104-1115 (2016).

15. Lee, K. H. et al. PLoS One 14, e0207898 (2019).

Competing interests

The authors declare no competing interests.

\title{
Is VITT really a HIT
}

Thrombosis complicates SARS-CoV-2 infection and vaccination. Recent data are being used to identify the autoimmune antibody repertoires responsible for the excessive activation of coagulation and platelets.

\section{Zaverio M. Ruggeri and Wolfram Ruf}

$\mathrm{n}$ a recent Nature article, Huynh et al. ${ }^{1}$ identify the epitope on platelet factor 4 (PF4; also known as CXCL4) recognized by five antibodies isolated from people who developed thrombocytopenia and thrombosis after receiving the ChAdOx1 nCoV-19 vaccine against COVID-19 (commonly referred to as the 'University of Oxford-AstraZeneca' vaccine) $)^{2,3}$. This syndrome, which is characterized by thrombosis in unusual sites (notably, sinus veins of the brain), has an incidence of one case or less per 100,000 vaccinations, a female/male ratio of $\sim 2: 1$ and a mortality of about three in ten cases. Greinacher et al. concluded that platelet-activating anti-PF4 antibodies, akin to heparin-induced thrombocytopenia (HIT), cause this new syndrome, for which they propose the name 'vaccine-induced immune thrombotic thrombocytopenia' (VITT) ${ }^{3}$. As none of the patients with VITT had received heparin during the time required for the appearance of symptoms, the authors suggested that VITT resembles the previously described autoimmune HIT, in which thrombotic thrombocytopenia is heparin independent ${ }^{4}$.

HIT is phenotypically diverse, but always involves IgG antibodies that react with PF4, a positively charged tetrameric protein (polycationic) of the CXC chemokine family stored in platelet $\alpha$-granules and released in response to platelet activation. Typical antibodies involved in HIT react with PF4 bound to negatively charged heparin polymers (polyanionic) that form ultra-large pathogenic immunocomplexes and activate platelets through the receptor Fc $\gamma$ RIIA $(\mathrm{CD} 32 \mathrm{a})^{5}$. By contrast, HIT anti-PF4 autoimmune antibodies directly cluster PF4 tetramers and thereby activate platelets without heparin. Thus, a multiplicity of factors that affect size and stability of anti-PF4 immunocomplexes can explain the pathogenesis and phenotypic diversity of HIT. Of particular importance is the binding affinity of anti-PF4 antibodies, but equally relevant are the electrostatic interactions of polycationic PF4 with polyanions that, in addition to exogenous heparin, might include glycosaminoglycans and polyphosphates associated with platelets or other vascular and tissue cells. Therefore, organ-specific characteristics and individual platelet phenotypes can affect the assembly, size and physicochemical properties of anti-PF4-PF4 antigenantibody complexes. Then, engagement of Fc $\gamma$ RIIA leads to enhanced procoagulant activity with increased thrombin generation, fibrin deposition and thrombin feedback regulation of platelet activation, which creates a self-perpetuating highly prothrombotic environment in the vessel lumen and on cell surfaces (Fig. 1). The end result is the syndrome of thrombocytopenia caused by platelet consumption and immunothrombosis, in which anti-PF4 IgG antibodies are common to HIT and VITT.

The study by Huynh et al provides new insights into pathogenic anti-PF4 antibodies by identifying the epitope specificity of five VITT antibodies and ten HIT antibodies, with four of the latter being heparin dependent $^{1}$. The results of testing 70 PF4 mutants, each with a single residue replaced with alanine, establish that the epitope common to all VITT antibodies, but in none of the HIT antibodies, includes four residues shared with the heparin-binding site of PF4. VITT and HIT anti-PF4 antibodies, whether heparin dependent or independent, have similar binding characteristics, which suggests a comparable ability to form platelet-activating immunocomplexes. These data indicate that structural characteristics of anti-PF4 antibody epitopes might explain pathogenicity, which delineates a method for characterizing antibodies linked to well-defined disease phenotypes.

The origin and development of VITT autoantibodies in the context of immunization remains an open question, although much of what is known about the origin of HIT antibodies might apply to VITT ${ }^{4,6}$. The delayed onset of the syndrome would indicate that VITT anti-PF4 antibodies undergo expansion after the initial vaccination-associated 


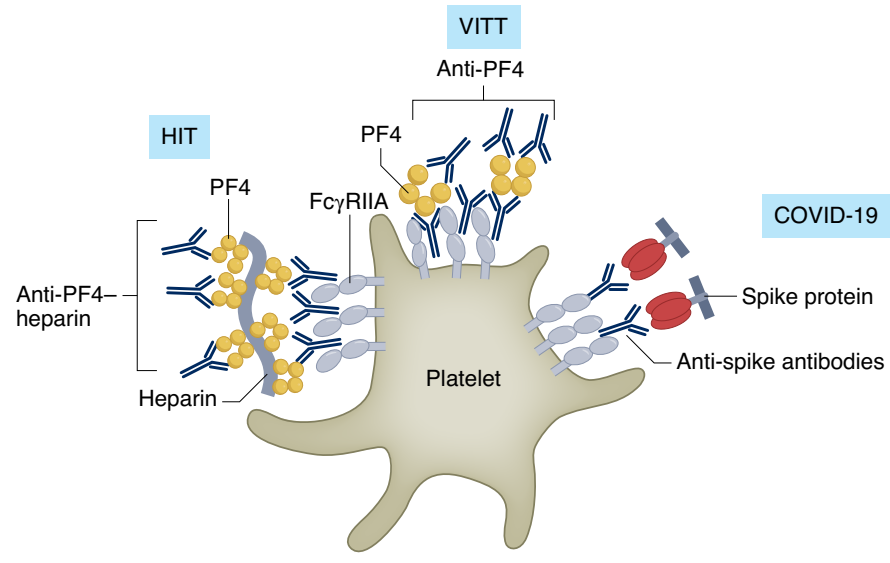

b

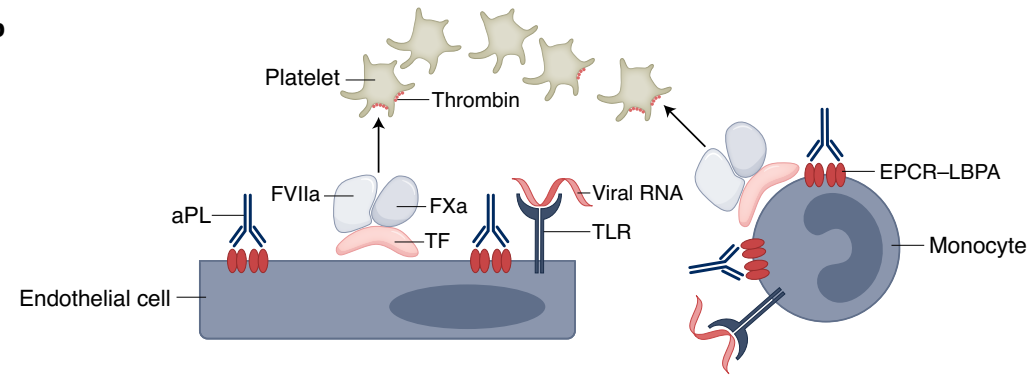

Fig. 1 | Platelet activation in HIT, VITT and COVID-19. a, Antibodies to PF4 in VITT and HIT, as well as antibodies to the SARS-CoV-2 spike protein in patients with COVID-19, activate platelets through FcyRIIA signaling induced by aggregated IgG antibodies within immunocomplexes. b. Direct platelet activation though FcyRIIA can be amplified in severe COVID-19 disease through the activation of coagulation by the convergence of viral pathogen-sensing receptors and autoimmune aPL on monocytes and endothelial cells, which in turn causes thrombin generation. TF, tissue factor; TLR, Toll-like receptor; EPCR-LBPA, lysobisphosphatidic acid bound to endothelial protein $C$ receptor.

immune reaction. Notably,

immunocomplex-dependent platelet activation mediated by Fc $\gamma$ RIIA has also been documented in patients with COVID-197. Moreover, aberrant glycosylation of immunocomplexes directed against the coronavirus SARS-CoV-2 spike protein promotes exacerbated platelet activation that involves Fc receptor signaling ${ }^{8}$, which indicates a COVID-19-specific immune mechanism to promote platelet hyper-reactivity. Unlike VITT, the severe coagulopathy observed in hospitalized patients with COVID-19 is more complex and involves immune mechanisms of platelet and coagulation activation specifically associated with SARS-CoV-2. Vaccination against COVID-19 might, in principle, elicit innate immune signaling that overlaps that induced by infection with SARS-CoV-2. However, the coagulation activation in severely ill hospitalized patients is characterized by markedly increased plasma concentrations of the D-dimer fibrin-degradation product coupled with inflammatory markers ${ }^{9}$. This phenotype, which indicates multiple reciprocal connections between inflammation induced by infection with SARS-CoV-2 and coagulation, is distinct from adverse effects that are linked to vaccinations.

The specific immune response to infection with SARS-CoV-2 depends on $B$ cells with a near-germline repertoire of virus-specific antibodies and leads to the development of autoantibodies to several immunoregulatory antigens ${ }^{10}$. Viral infections are associated frequently with expansion of anti-phospholipid antibodies (aPL), which can also develop transiently in adenoviral immunization protocols ${ }^{11}$. The severity of COVID-19 correlates with the development of $\mathrm{aPL}^{9}$ that specifically recognize the endosomal lipid lysobisphosphatidic acid bound to the endothelial protein $C$ receptor, a cell surface antigen linked to the development of autoimmune disease ${ }^{12}$. By contrast, although the genes encoding aPL hypermutate in autoimmune disease and the resultant aPL acquire cross-reactivity with other coagulation and immunoregulatory plasma proteins (prothrombin and $\beta 2$ glycoprotein I), the expansion of cross-reactive aPL is infrequent in patients with COVID-19 ${ }^{13}$. In the latter, aPL of the IgG subtype are produced by innate-like B-1 cells distinct from the extrafollicular B cell population linked to the specific anti-SARS-CoV-2 response $^{14}$. Lacking the autoimmune aPL that react with the immunoregulatory $\beta 2$ glycoprotein I to activate platelets, pathogenic aPL in COVID-19 probably induce coagulation through upregulation of the coagulation-initiator tissue factor on monocytes and endothelial cells, which are involved in COVID-19-associated coagulopathy ${ }^{9}$. Thus, the restricted reactivity of anti-PF4 antibodies in VITT in the context of immunization contrasts with a diverse autoantibody response in severe COVID-19 that induces a variety of platelet, endothelial and monocyte responses. These latest studies of thrombosis in COVID19 disease and of the complications of vaccination against SARS-CoV-2 have identified new connections between antiviral immune responses and the development of autoimmune antibodies that warrant further investigation.

\section{Zaverio M. Ruggeri(D) ${ }^{1 \times}$ and Wolfram Ruf (D) 2,3凶 \\ ${ }^{1}$ Department of Molecular Medicine, MERU-Roon Research Center on Vascular Biology, The Scripps Research Institute, La Jolla, CA, USA. ${ }^{2}$ Department of Immunology and Microbiology, The Scripps Research Institute, La Jolla, CA, USA. ${ }^{3}$ Center for Thrombosis and Hemostasis, Johannes Gutenberg University Medical Center, Mainz, Germany. \\ $\bigotimes_{e \text {-mail:ruggeri@scripps.edu; ruf@uni-mainz.de }}$}

Published online: 22 October 2021

https://doi.org/10.1038/s41590-021-01042-9

References

1. Huynh, A., Kelton, J. G., Arnold, D. M., Daka, M. \& Nazy, I. Nature 596, 565-569 (2021).

2. Schultz, N. H. et al. N. Engl. J. Med. 384, 2124-2130 (2021).

3. Greinacher, A. et al. N. Engl. J. Med. 384, 2092-2101 (2021).

4. Greinacher, A., Selleng, K. \& Warkentin, T. E. J. Thromb. Haemost. 15, 2099-2114 (2017).

5. Arman, M. \& Krauel, K. J. Thromb. Haemost. 13, 893-908 (2015).

6. Arepally, G. M. Blood 129, 2864-2872 (2017).

7. Althaus, K. et al. Blood 137, 1061-1071 (2021).

8. Bye, A. P. et al. Blood https://doi.org/10.1182/blood.2021011871 (2021).

9. Hollerbach, A. et al. J. Thromb. Haemost. 19, 2335-2347 (2021).

10. Wang, E. Y. et al. Nature 595, 283-288 (2021).

11. Crank, M. C. et al. PLoS One 11, e0166393 (2016).

12. Müller-Calleja, N. et al. Science 371, eabc0956 (2021).

13. Zuo, Y. et al. Sci. Transl. Med. 12, eabd3876 (2020).

14. Woodruff, M. C. et al. Nat. Immunol. 21, 1506-1516 (2020).

\section{Competing interests}

Z.M.R. is the founder, president and CEO of MERU VasImmune (a company that develops coagulation tests). W.R. is affiliated with MERUVasImmune and reports intellectual property and royalty income from ARCA biopharma (for the treatment of COVID-19-associated coagulopathy). 